\title{
Role of Open Channels in Overlapping Resonances Studied by Multichannel Quantum Defect Theory in Systems Involving 2 Nondegenerate Closed and Many Open Channels
}

\author{
Chun-Woo Lee \\ Department of Chemistry, Ajou University, Woncheon-Dong, Yeongtong-Gu, Suwon 443-749, Korea \\ E-mail: clee@ajou.ac.kr
}

Received August 17, 2010, Accepted September 7, 2010

\begin{abstract}
Previous work on the phase-shifted version of the multichannel quantum-defect theory (MQDT) for a system involving 2 closed and many open channels (Lee, C.-W. Bull. Korean Chem. Soc. 2010, 31, 1669) was extended to obtain the formulae of the spectral shape parameters with the structure of a pole extracted explicitly for general cases only limited by 2 non-degenerate closed channels. The theory was applied to the narrow $6 \mathrm{p}_{1 / 2,3 / 2} n \mathrm{p} J=1$ autoionizing Rydberg series in barium perturbed by the $6 \mathrm{p}_{3 / 2} n \mathrm{f}$ states obtained by de Graaff et al.
\end{abstract}

Key Words: Phase-shifted MQDT, Overlapping resonance, $q$ reversal, Effective continuum, Autoionizing Rydberg series

\section{Introduction}

A Rydberg series perturbed by an interloper is an important system showing complex resonance phenomena due to the overlapping resonance. Multichannel quantum defect theories (MQDT), with the implementation of phase renormalization, are extremely powerful for dealing with the observed overlapping resonances. ${ }^{1-5}$ A system including only 3 channel systems with one open and 2 closed chosen as the model system was studied extensively by Giusti-Suzor and Lefebvre-Brion, Cooke and Cromer, Wintgen and Friedrich and Ueda. ${ }^{3,4,6,7}$ They investigated the phenomena in complex resonances, such as intensity borrowing, $q$ reversals and truly bound states in a continuum. Among these studies, the reformulation of the phase-shifted MQDT by Ueda to disentangle the interloper spectra from the perturbed autoionizing Rydberg series provides a very useful tool for analyzing overlapping resonance spectra, and was instrumental in Refs. [8] and [9] for determining the mutual influences between an interloper and the autoionizing Rydberg series perturbed by it in the photoionization cross section $\sigma$ :

$$
\begin{aligned}
\sigma & =\tilde{\sigma}_{0} \frac{\left(\tilde{\varepsilon}_{2}+\tilde{q}_{2}\right)^{2}}{\tilde{\varepsilon}_{2}^{2}+1} \frac{\left(\tilde{\varepsilon}_{\text {leff }}+\tilde{q}_{1 \mathrm{eff}}\right)^{2}}{\tilde{\varepsilon}_{\text {leff }}^{2}+1} \\
& =\tilde{\sigma}_{\text {interloper }} \frac{\left(\tilde{\varepsilon}_{\text {leff }}+\tilde{q}_{\text {leff }}\right)^{2}}{\tilde{\varepsilon}_{\text {leff }}^{2}+1}
\end{aligned}
$$

where index 2 denotes the Rydberg series of an interloper, 1 for the perturbed Rydberg series, $\tilde{\varepsilon}_{2}$ stands for the reduced energy of the Rydberg series $2, \tilde{q}_{2}$ is the line shape index and $\tilde{\varepsilon}_{\text {leff }}$ and $\tilde{q}_{1 \text { eff }}$ are the effective reduced energy and line shape index for the perturbed Rydberg series 1, respectively. Eq. (1) shows that the effect of an interloper to the autoionizing spectra of a Rydberg series appears in two ways. One is to modulate the entire shape of the spectra so that its envelope is given by the autoionization spectra

$$
\tilde{\sigma}_{\text {Interloper }}=\tilde{\sigma}_{0}\left(\tilde{\varepsilon}_{2}+\tilde{q}_{2}\right)^{2} /\left(\tilde{\varepsilon}_{2}^{2}+1\right)
$$

of the interloper and the other is to perturb each resonance peak of the spectra of the Rydberg series with its effect incorporated into the resonance parameters of each resonance peak so that $\tilde{\varepsilon}_{1}$ and $\tilde{q}_{1}$ are replaced by the effective $\tilde{\varepsilon}_{\text {leff }}$ and $\tilde{q}_{\text {leff }}$, respectively. The perturbation is local so that $\tilde{q}_{\text {leff }}\left(\tilde{\varepsilon}_{2}\right)$ is no longer a constant function of the energy $\tilde{\varepsilon}_{2}$, which is in contrast to the constant $\tilde{q}_{1}$ for the unperturbed autoionizing Rydberg series 1 .

Ref. [8] reported that the autoionization cross section $\sigma$ of (1) is dominated by the singular behavior of $\left(\tilde{\varepsilon}_{\text {leff }}+\tilde{q}_{\text {leff }}\right)^{2} /$ $\left(\tilde{\varepsilon}_{\text {leff }}^{2}+1\right)$ of the perturbed series induced by the pole structures of $\tilde{q}_{\text {leff }}$

$$
\begin{aligned}
\tilde{q}_{\text {leff }}=\tilde{q}_{1} & -\left(1+\tilde{q}_{2}^{2}\right) \frac{\tilde{q}_{1}-\tilde{q}_{\mathrm{W}}}{\tilde{q}_{2}-\tilde{q}_{\mathrm{W}}} \frac{1}{\tilde{\varepsilon}_{2}+\tilde{q}_{2}} \\
& -\left(1+\tilde{q}_{\mathrm{W}}^{2}\right) \frac{\tilde{q}_{1}-\tilde{q}_{2}}{\tilde{q}_{\mathrm{W}}-\tilde{q}_{2}} \frac{1}{\tilde{\varepsilon}_{2}+\tilde{q}_{\mathrm{W}}}
\end{aligned}
$$

at $\tilde{\varepsilon}_{2}=-\tilde{q}_{2}$ and $-\tilde{q}_{W}$ and $q$ reversals occurring at the zero surface structures of the line profile parameter $\tilde{q}_{\text {leff }}$ given by

$$
\tilde{q}_{1 \mathrm{eff}}\left(\tilde{\varepsilon}_{2}\right)=\tilde{q}_{1} \tilde{\varepsilon}_{2}^{2}-\left(1-\tilde{q}_{2} \tilde{q}_{\mathrm{W}}\right) \tilde{\varepsilon}_{2}+\tilde{q}_{1}-\tilde{q}_{2}-\tilde{q}_{\mathrm{W}}=0
$$

where $\tilde{q}_{\mathrm{W}}$ is another line shape parameter pertaining to the perturbed line width $\tilde{W}_{\text {leff }}$ of series 1 by the interloper. Such pole and zero surface structures were incorporated into the form of a map to follow the change and control the patterns of the autoionization spectra in previous work. ${ }^{8}$

Ueda's formalism ${ }^{7}$ was extended further to systems involving more than 1 open channel to determine the role of the additional open channels on channel coupling and the spectra, which might be viewed as a controversial field in MQDT with unconfirmed 
assertions remaining to be resolved. Fano and Cooper reported that a discrete state can interact with only one type of continuum. Mies developed their theory further and proposed the use an overlapping matrix to represent the extent of overlap when a complete superimposition of overlapping resonances is no longer obtained when more than 1 open channel is involved in the configuration mixing $(\mathrm{CM})$ theory. Mies's $\mathrm{CM}$ theory was implemented into MQDT to treat the spectral width in the isolated core excitation (ICE) spectra by Lecomte. ${ }^{10}$ Cohen challenged this problem of handling the channel coupling induced by additional open channels in the MQDT formulation with the approximate analytic solution but was only partially successful. ${ }^{11}$ The author's previous work applied Ueda's formalism for this system involving more than 1 open channel, and successfully dealt with the Martins and Zimmermann's photoionization spec$\operatorname{tra}^{12}$ of the Rydberg series $\mathrm{Cu}$ I $3 \mathrm{~d}^{9} 4 \mathrm{~s}\left({ }^{1} \mathrm{D}_{2}\right) n \mathrm{~d}^{2} \mathrm{G}_{9 / 2}$ perturbed by the interloper, $3 d^{9} 4 p^{24} F_{9 / 2}$, for which Cohen's 4 channel QDT failed. ${ }^{11}$ However, this work was unsuccessful in obtaining explicit formulae of the zero surfaces of the line profile index $\tilde{q}_{\text {leff }}$ for general systems limited only by 2 non-degenerate closed channels. This paper reports the formulae with the structure of a pole explicitly extracted for such general systems. Obtaining explicit formulae is important for deriving the physical terms that determine the spectral shape and obtaining the entire spectrum of possible patterns of the spectral shape. The formulae was applied to the $\mathrm{Ba} 6 \mathrm{p}_{1 / 2} n \mathrm{p}_{3 / 2} J=1$ autoionizing Rydberg series with $6 \mathrm{p}_{3 / 2} n \mathrm{f}$ series as an interloper

\section{Overview}

In the presence of more than one open channel, the total autoionization cross section is given by a sum of the cross sections over all open channels. For practical purposes, it is better to use the eigenchannels of the physical scattering matrices $\mathbf{S}$ in place of the open channels. If index $\rho$ is used for the eigenchannels, the total autoionization cross section is given by the sum over $\rho$ as follows:

$$
\sigma=\sum_{\rho} \sigma_{\rho}=\frac{4 \pi^{2} \alpha \omega}{3} \sum_{\rho}\left|\tilde{\mathbf{D}}_{\rho}\right|^{2}
$$

where $\alpha$ denotes the fine structure constant, $\omega$ is the frequency of light and $\tilde{D}_{\rho}$ is the transition dipole moment to the eigenchannel wavefunction $\tilde{\mathbf{\Psi}}_{\rho}$. Cohen reported that among the eigenchannels, only one shows most of the resonance behavior and the contributions of remaining ones can be treated as backgrounds to the autoionization spectra: $\sigma=\sigma_{\text {res }}+\sigma_{\text {nr }}$ with $\sigma_{\mathrm{nr}}=\Sigma_{\rho \neq \mathrm{res}} \sigma_{\rho} .{ }^{11}$ For the resonance cross section, Ueda's type of decoupling can be applied as follows: ${ }^{9}$

$$
\sigma_{\text {res }}=K \frac{\left(\tilde{\varepsilon}_{2}+\tilde{q}_{2 \mathrm{res}}\right)^{2}}{\tilde{\varepsilon}_{2}^{2}+1} \frac{\left(\tilde{\varepsilon}_{1 \mathrm{eff}}+\tilde{q}_{1 \mathrm{reseff}}\right)^{2}}{\tilde{\varepsilon}_{\text {leff }}^{2}+1}
$$

where $K$ denotes $\left(4 \pi^{2} \alpha \omega / 3\right) \tilde{d}_{\text {res }}^{2} A_{\text {res }}^{2}$ with the transition dipole moment $\tilde{d}_{\text {res }}$ to the resonance eigenchannel and $A_{\text {res }}$ denotes the avoided crossing term. Refs. [8] and [9] showed that the de- coupling (6) of the interloper's spectrum from the perturbed autoionizing Rydberg series can be made more powerful by exploiting the pole and zero surface structures of the line asymmetry parameter $\tilde{q}_{\text {leff }}$, such as (3) and (4). Attempts to find similar explicit pole structures from (6) for systems involving more than 1 open channel were unsuccessful in previous work. Partial success was obtained for special cases of a negligible direct interaction between the closed channels and a negligible transition to the open channels. This paper reports the explicit formula for the general cases. Before deriving this general explicit formula, let us describe the system for which the present theory was developed.

Brief introduction of MQDT formulation. Consider the photoionization energy range satisfying $I_{3}<\cdots<I_{n}<E<I_{1}<I_{2}$ so that channels 1 and 2 are closed to direct ionization, and channels from 3 to $n$ are open to ionization. In this case, Rydberg series 1 and 2 converging to the respective ionization limit, $I_{1}$ and $I_{2}$, produce autoionizing photoionization spectra by channel coupling with the open channels from 3 to $n$. In this system satisfying $I_{1}<I_{2}$, the autoionizing lines of Rydberg series 2 act as interlopers for denser autoionizing lines of Rydberg series 1 and produce complex overlapping resonances. Multichannel quantum defect theories (MQDT) used to deal with these overlapping resonances utilize a few channel interaction parameters represented by the reactance matrix elements $K_{i j}$ for coupling between channels $i$ and $j$. $^{2}$ The phase-shifted version of MQDT is normally employed to take advantage of the minimal set of non-zero reactance matrix elements given by

$$
\tilde{K}=\left(\begin{array}{cc}
\tilde{K}^{c c} & \tilde{K}^{c o} \\
\tilde{K}^{o c} & \tilde{K}^{o o}
\end{array}\right)=\left(\begin{array}{ccccc}
0 & \tilde{K}_{12} & \tilde{K}_{13} & \cdots & \tilde{K}_{1 n} \\
\tilde{K}_{12} & 0 & \tilde{K}_{23} & \cdots & \tilde{K}_{2 n} \\
\tilde{K}_{13} & \tilde{K}_{23} & 0 & \cdots & 0 \\
\vdots & \vdots & \vdots & \ddots & \vdots \\
\tilde{K}_{1 n} & \tilde{K}_{2 n} & 0 & \cdots & 0
\end{array}\right)
$$

for the present system. ${ }^{10}$ Let $\tilde{\beta}_{i}$ denote $\pi\left(v_{i}+\mu_{i}\right)$ where the effective quantum number $v_{i}$ is defined by $E=I_{j}-\mathrm{Ryd} / v_{j}^{2}$, with Rydberg constant Ryd for the ionization threshold energy $I_{i}$. Then, the above-mentioned eigenchannel wavefunctions $\tilde{\mathbf{\Psi}}_{\rho}$ which are the eigenfunctions of the physical reactance matrix $\tilde{\mathbf{K}}$ are given in terms of the submatrices of $\tilde{K}$ in Eq. (7) as $\tilde{K}^{o c}\left(\tan \tilde{\beta}+\tilde{K}^{c c}\right)^{-1} \tilde{K}^{c o}$ and are normally expanded in terms of the real standing-wave channel basis functions in the open channel space $P$ and closed channel space $Q$ as follows:

$$
\tilde{\boldsymbol{\Psi}}_{\rho}=\sum_{i \in P} \tilde{\Psi}_{i} \tilde{Z}_{i \rho} \cos \tilde{\delta}_{\rho}+\sum_{i \in Q} \tilde{\Psi}_{i} \cos \pi\left(v_{i}+\mu_{i}\right) \tilde{Z}_{i \rho}
$$

In the expansion, the additional factors, $\cos \tilde{\delta}_{\rho}$ and $\cos \pi$ $\left(v_{i}+\mu_{i}\right)$, to the expansion coefficients are introduced to make $\tilde{Z}_{i \rho}(i \in P)$ unitary and essential because the standing-waves $\tilde{\Psi}_{i}$ defined outside the reaction zone as

$$
\tilde{\Psi}_{i}=\sum_{j} \Phi_{j}(\omega)\left[\tilde{f}_{j}(R) \delta_{j i}-\tilde{g}_{j}(R) \tilde{K}_{j i}\right], \quad\left(R>R_{0}\right)
$$


with the phase-shifted regular and irregular base pair ${ }^{3}\left(\tilde{f}_{j}, \tilde{g}_{j}\right)$

$$
\begin{aligned}
& \tilde{f}_{j}=f_{j} \cos \pi \mu_{j}-g_{j} \sin \pi \mu_{j} \\
& \tilde{g}_{j}=g_{j} \cos \pi \mu_{j}+f_{j} \sin \pi \mu_{j}
\end{aligned}
$$

are not energy-normalized. The regular and irregular base pair $\left(f_{j}, g_{j}\right)$ in $(10)$ belongs to the $j^{\text {th }}$ ionization threshold energy $I_{j}$. As is well known, ${ }^{2}$ the expansion coefficients into the closed channel basis functions in (8) can be obtained as follows:

$$
\cos \tilde{\beta} \tilde{Z}_{i \rho}^{\mathrm{c}}=-\left(\tan \tilde{\beta}+\tilde{K}^{\mathrm{cc}}\right)^{-1} \tilde{K}^{\mathrm{co}} \tilde{Z}_{\rho}^{\mathrm{c}} \cos \tilde{\delta}_{\rho},
$$

where $\tilde{\beta}_{i}$ is $\pi\left(v_{i}+\mu_{i}\right)$ and $\tilde{\delta}_{\rho}(\rho \in P)$ is the eigenphase shift obtained from the compatibility equation

$$
\operatorname{det}\left(\tilde{\mathbf{K}}-\tan \tilde{\delta}_{\rho}\right)=0
$$

of the MQDT equation

$$
\left(\begin{array}{cc}
\tilde{K}^{\mathrm{cc}}+\tan \tilde{\beta} & \tilde{K}^{\mathrm{co}} \\
\tilde{K}^{\mathrm{oc}} & -\tan \tilde{\delta}_{\rho}
\end{array}\right)\left(\begin{array}{c}
\tilde{Z}_{\rho}^{\mathrm{c}} \\
\tilde{Z}_{\rho}^{\mathrm{o}}
\end{array}\right)=0
$$

obtained by setting the coefficients of the exponentially increasing terms of (8) to zero.

Previous results relevant to the present formulation. As stated above, only one eigenchannel dominates the resonance behavior and can be regarded as a resonance eigenchannel, ${ }^{11}$ indicating that the parameters of the resonance dominant eigenchannel can be used as resonance parameters. However, a better way to obtain the resonance parameters is to consider the sum $\Sigma_{\rho} \tilde{\delta}_{\rho}$ of the eigenphase shifts that remove the avoided crossing interactions analytically: ${ }^{13}$

$$
\begin{aligned}
\operatorname{det}(\tilde{\boldsymbol{S}}) & =\exp \left(-2 i \sum_{\rho} \tilde{\delta}_{\rho}\right)=\frac{\operatorname{det}\left(\tan \tilde{\beta}+\tilde{\boldsymbol{\kappa}}^{\mathrm{cc} *}\right)}{\operatorname{det}\left(\tan \tilde{\beta}+\tilde{\boldsymbol{\kappa}}^{\mathrm{cc}}\right)} \\
& =\exp \left(-2 i \tilde{\delta}_{r}\right),
\end{aligned}
$$

where $\tilde{\kappa}^{\mathrm{cc}}$ denotes $\tilde{K}^{\mathrm{cc}}-i \tilde{K}^{\mathrm{co}}\left(1+i \tilde{K}^{\mathrm{oo}}\right)^{-1} \tilde{K}^{\mathrm{oc}}=\tilde{K}^{\mathrm{cc}}-i \tilde{K}^{\mathrm{co}} \tilde{K}^{\mathrm{oc}} \cdot{ }^{10}$ $\Sigma_{\rho} \tilde{\delta}_{\rho}^{0}=0$ from $\tilde{K}^{\text {oo }}=0$ in the phase-shifted MQDT yields the $3^{\text {rd }}$ equality in Eq. (14). From (14), the resonant phase shift $\tilde{\delta}_{r}$ is equivalent to the phase of $\operatorname{det}\left(\tan \tilde{\beta}+\tilde{\kappa}^{\mathrm{cc}}\right)$ and the square of the modulus of $\operatorname{det}\left(\tan \tilde{\beta}+\tilde{\kappa}^{\text {cc }}\right)$ was obtained as follows: ${ }^{9}$

$$
C^{2}=\tilde{W}_{\text {leff }}^{2} \tilde{W}_{2}^{2}\left(\tilde{\varepsilon}_{\text {leff }}^{2}+1\right)\left(\tilde{\varepsilon}_{2}^{2}+1\right)
$$

where $\tilde{\varepsilon}_{i}$ denotes $\tan \tilde{\beta}_{i} / \tilde{W}_{i}(i=1,2)$ and $\tilde{W}_{i}$ denotes $\tilde{\xi}_{i}^{2}$, and stands for the reduced energy and spectral width of the Rydberg series $i$, respectively. The effective reduced spectral width and energy $\tilde{W}_{\text {leff }}$ and $\tilde{\varepsilon}_{\text {leff }}$ for perturbed Rydberg series 1 were obtained as follows:

$$
\begin{aligned}
& \tilde{W}_{\text {leff }}=\tilde{W}_{1}\left[\sin ^{2} \theta+\cos ^{2} \theta \frac{\left(\tilde{\varepsilon}_{2}+\tilde{q}_{\mathrm{W}}\right)^{2}}{\tilde{\varepsilon}_{2}^{2}+1}\right] \\
& \tilde{\varepsilon}_{\text {leff }}=\frac{\tilde{W}_{1}}{\tilde{W}_{\text {leff }}}\left[\tilde{\varepsilon}_{1}+\cos ^{2} \theta \frac{\tilde{\varepsilon}_{2}\left(1-\tilde{q}_{\mathrm{W}}^{2}\right)+2 \tilde{q}_{\mathrm{W}}}{\tilde{\varepsilon}_{2}^{2}+1}\right],
\end{aligned}
$$

where $\tilde{q}_{\mathrm{W}}$ is also the generalization of $-\tilde{K}_{12} /\left(\tilde{\xi}_{1} \tilde{\xi}_{2}\right)$ in the 3 channel QDT involving 1 open and 2 closed channels and is defined as follows: ${ }^{9}$

$$
\tilde{q}_{\mathrm{W}}=-\frac{\tilde{K}_{12}}{\tilde{\xi}_{1} \cdot \tilde{\xi}_{2}}=-\frac{\tilde{K}_{12}}{\tilde{\xi}_{1} \tilde{\xi}_{2} \cos \theta} .
$$

$\tilde{\xi}_{i}$ denotes the modulus of the column vector $\tilde{\xi}_{i}$, whose $k^{\text {th }}$ component is defined as $\tilde{K}_{k i}^{\text {oc }}$ and stands for the coupling strength of the closed channel $i$ with open channels. Similarly, $\tilde{\xi}_{1} \cdot \tilde{\xi}_{2}$ denotes the scalar product of two vectors in open channel space $P$ defined by $\Sigma_{k \in P} \tilde{K}_{k 1} \tilde{K}_{k 2}$ and stands for the indirect coupling between closed channels 1 and 2 . The reduced width (16) is the same as the scaled width used to extract the minimal number of parameters proposed by Lecomte when many open channels are involved in the autoionization spectra. ${ }^{10}$ In an analogy with $\tilde{\varepsilon}_{1}=-\cot \tilde{\delta}_{\text {rl }}, \tilde{\varepsilon}_{\text {leff }}$ satisfies the equation $\tilde{\varepsilon}_{\text {leff }}=-\cot \tilde{\delta}_{\text {rleff }}$, where $\tilde{\delta}_{\text {rleff }}$ is the resonance phase shift for the perturbed Rydberg series 1 and satisfies $\tilde{\delta}_{\mathrm{r}}=\tilde{\delta}_{\mathrm{rleff}}+\tilde{\delta}_{\mathrm{r} 2}$. See Appendix A for more details.

With (8) and (11), the transition dipole moment $\tilde{\mathbf{D}}_{\rho}[=$ $\left(\tilde{\Psi}_{\rho}|T| i\right)$ ] of Eq. (5) to eigenchannel $\rho$ was transformed into

$$
\tilde{\mathbf{D}}_{\rho}=\tilde{d}_{\rho} \tilde{W}_{2}\left(\tilde{\varepsilon}_{2}+\tilde{q}_{2 \rho}\right) \tilde{W}_{\text {leff }}\left(\tilde{\varepsilon}_{\text {leff }}+\tilde{q}_{\text {leff }}\right) \frac{A_{\rho}}{C},
$$

where $\tilde{d}_{\rho}$ denotes $\sum_{i \in P} \tilde{D}_{i} \tilde{Z}_{i \rho}^{o}, A_{\rho}$ stands for the avoided cross interaction and is close to unity, whose explicit form can be found in Ref. [9]. This will not be considered further as it does not play a significant role. The line profile parameters, $\tilde{q}_{1 \rho}$ and $\tilde{q}_{2 \rho}$, now depend on the eigenchannels and denote

$$
\begin{aligned}
& \tilde{q}_{1 \rho}=-\frac{\tilde{D}_{1}}{\tilde{d}_{\rho}} \frac{\tilde{\xi}_{1}^{T} \tilde{\mathbf{Z}}_{\rho}^{o}}{\tilde{\xi}_{1}^{T} \tilde{\xi}_{1}}=-\frac{\tilde{D}_{1}}{\tilde{d}_{\rho}^{(e)}} \frac{\cos \alpha_{\rho}}{\tilde{\xi}_{1}} \\
& \tilde{q}_{2 \rho}=-\frac{\tilde{D}_{2}}{\tilde{d}_{\rho}} \frac{\tilde{\xi}_{2}^{T} \tilde{\mathbf{Z}}_{\rho}^{o}}{\tilde{\xi}_{2}^{T} \tilde{\xi}_{2}}=-\frac{\tilde{D}_{2}}{\tilde{d}_{\rho}^{(e)}} \frac{\cos \beta_{\rho}}{\tilde{\xi}_{2}}
\end{aligned}
$$

where $\cos \alpha_{\rho}$ and $\cos \beta_{\rho}$ are the direction cosines of $\tilde{\mathbf{Z}}_{\rho}^{\mathrm{o}}$ of the unit length into the axes $\tilde{\xi}_{1}$ and $\tilde{\xi}_{2}$, respectively. The ratio of the direction cosines $\cos \beta_{\rho} / \cos \alpha_{\rho}$ gives the ratio of contributions of Rydberg series 1 and 2 in eigenchannel $\rho_{2}$ and will be denoted as $r_{\rho}$. Its other equation is $r_{\rho}=\left(\tilde{\xi}_{2 \rho} / \tilde{\xi}_{2}\right) /\left(\tilde{\xi}_{1 \rho} / \tilde{\xi}_{1}\right)$. The remaining parameter $\tilde{q}_{1 \rho \text { eff }}$ in (19) is the effective line shape parameter and was obtained as follows: 


$$
\begin{aligned}
\tilde{q}_{1 \rho \mathrm{eff}} & =\frac{\tilde{W}_{1}}{\tilde{W}_{1 \mathrm{eff}}}\left[\cos ^{2} \theta \frac{\tilde{\varepsilon}_{2}\left(\tilde{q}_{\mathrm{W}}^{2}-1\right)-2 \tilde{q}_{\mathrm{W}}}{\tilde{\varepsilon}_{2}^{2}+1}\right] \\
+ & \frac{\tilde{W}_{1}}{\tilde{W}_{\text {leff }}}\left[\tilde{q}_{1 \rho}-\frac{\left(\tilde{q}_{\mathrm{W}} \cos \theta-\tilde{q}_{1 \rho} r_{\rho}\right)\left(\tilde{q}_{\mathrm{W}} \cos \theta-\tilde{q}_{2 \rho} / r_{\rho}\right)}{\tilde{\varepsilon}_{2}+\tilde{q}_{2 \rho}}\right] .
\end{aligned}
$$

With the substitution of (15), (19) gives the autoionization cross section formula, which takes the form of (6) when $\rho=$ res.

Note that the decomposition of the autoionization cross section for the systems involving 2 closed and more than 1 open channel relies on the dominance of the total cross sections by the resonant eigenchannel. ${ }^{11}$ Such decomposition would be exact if the dynamics at the excited states alone are considered, indicating that the decomposition is a more fundamental nature of the overlapping resonances. This fact derives from the exact isolation of the resonance phase shift $\tilde{\delta}_{\text {r }}$ into $\tilde{\delta}_{\text {rleff }}+\tilde{\delta}_{\text {r2 }}$, even when more than 1 open channel is involved. See Appendix A for proof.

Derivation of the general explicit formulae of $\tilde{\boldsymbol{q}}_{1 \text { eff. Adding }}$ additional open channels to the formulation is troublesome in the MQDT formulation. ${ }^{11}$ One of the problems is caused by the connection problem of the eigenchannels at different energies. To classify the eigenchannels into resonance and non-resonance ones, the continuity of the first derivative of the dynamic quantities, such as the eigenphase shifts $\tilde{\delta}_{\rho}$ needs to be sacrificed. This means that the dynamic quantities under this connection might not have continuous first derivatives because the connection is not based on achieving smooth variations in the physical quantities as a function of energy but simply sorting into resonance and non-resonance parts. Kinks caused by this discontinuity are not induced by real physics but by connection problems. Their removal will greatly simplify the investigation without losing any validity of the analysis associated with $q$ reversals. One way of removing the kink is to use the average value. The other way is to use solutions at the most remote points from the kinks. $\tilde{\varepsilon}_{1}=\infty$ or $\tan \tilde{\beta}_{1}=\infty$ corresponds to such a point that was already utilized in Ref. [9]. The analytical solution for the eigenchannel problem of $\underset{\tilde{\mathbf{K}}}{\tilde{\tilde{Z}}} \tilde{\kappa}_{\text {res }}=\tan \tilde{\delta}_{\text {res }} \tilde{Z}_{k \text { res }} \tilde{Z}_{\text {at }}$ this point can be given by $Z_{k \text { res }}=\tilde{K}_{2 k} / \tilde{\xi}_{2} \equiv \tilde{\xi}_{2 k} / \tilde{\xi}_{2}$ where $\tilde{Z}_{k \text { res }}$ denotes the $k^{\text {th }}$ component of the resonance eigenchannel (see Appendix $\mathrm{B}$ for proof when more than 2 open channels are involved). Note that this solution corresponds to the effective continuum ${ }^{4,14}$ or Fano's a state ${ }^{15}$ for autoionizing Rydberg series 2 acting as an interloper. This suggests that the points chosen to remove the kinks regard the interloper as a structured background for the perturbed autoionizing series. The line profile index $\tilde{q}_{\text {leff }}^{\text {res }}$ has no more constant. Its energy variation follows the structured background provided by the interloper.

Consider obtaining the resonant line profile index $\tilde{q}_{\text {lreseff }}$ ( $\rho=$ res ) for the perturbed dense Rydberg autoionizing series. Let us start by obtaining the solution-specific 3 parameters $r_{\rho=\mathrm{res}}, \tilde{q}_{1 \mathrm{res}}$ and $\tilde{q}_{2 \mathrm{res}}$ at $\tilde{\tilde{\varepsilon}}_{1} \rightarrow \infty$. First consider obtaining $r_{\text {res }}$ defined by $\left(\tilde{\xi}_{2 \text { res }} / \tilde{\xi}_{2}\right) /\left(\tilde{\xi}_{\text {1res }} / \tilde{\xi}_{1}\right)$. Because

$$
\begin{aligned}
\tilde{\xi}_{2 \text { res }} & =\tilde{\xi}_{2} \cdot \tilde{\mathbf{Z}}^{0}=\tilde{\xi}_{2} \cdot\left(\tilde{\xi}_{2} / \tilde{\xi}_{2}\right)=\tilde{\xi}_{2} \\
\tilde{\xi}_{\text {res }} & =\tilde{\xi}_{1} \cdot \tilde{\mathbf{Z}}^{0}=\tilde{\xi}_{1} \cos \theta
\end{aligned}
$$

$r_{\text {res }}$ can be obtained as

$$
r_{\text {res }}=\cos ^{-1} \theta \text {. }
$$

The other 2 parameters become

$$
\begin{aligned}
& \tilde{q}_{\text {1res }}=-\frac{\tilde{D}_{1} \cos \theta}{\tilde{d}_{\text {res }} \tilde{\xi}_{1}} \\
& \tilde{q}_{2 \text { res }}=-\frac{\tilde{D}_{2}}{\tilde{d}_{\text {res }} \tilde{\xi}_{2}}
\end{aligned}
$$

where $\tilde{d}_{\text {res }}$ is given by $\mathbf{D}^{\circ} \cdot\left(\tilde{\xi}_{2} / \tilde{\xi}_{2}\right)$. If $\theta=0$ and $\tilde{d}_{\text {res }}$ is regarded as $\tilde{D}_{3}$ in the 3 channel QDT, $\tilde{q}_{1 \text { res }}$ and $\tilde{q}_{2 \text { res }}$ in $(23)$ have the same forms as those of $\tilde{q}_{1}$ and $\tilde{q}_{2}$ in the 3 channel QDT, respectively.

Consider $\tilde{q}_{1 \rho \text { eff }}$ for $\rho=$ res . Note that only the second term of the right side of (21) is solution-specific. Denote the bracket part of the second term as $\tilde{q}_{1 \text { res }}^{\prime}$ and introduce $r$ and $t$ defined by $\tilde{D}_{1} \tilde{\xi}_{2} /\left(\tilde{D}_{2} \tilde{\xi}_{1}\right)$ and $\cos \theta / r$, respectively. Substitution of $\tilde{q}_{2 \text { res }} /$ $\tilde{q}_{1 \text { res }}=1 /(r \cos \theta)$ and $\cos \theta\left[r_{\text {res }}+q_{\text {2res }} /\left(q_{1 \text { res }} r_{\text {res }}\right)\right]=1+t$ from (22) and (23) transforms $q_{\text {1res }}^{\prime}$ into

$$
\tilde{q}_{1 \mathrm{res}}^{\prime}=\tilde{q}_{1 \mathrm{res}} \frac{\tilde{\varepsilon}_{2}-\tilde{q}_{\mathrm{W}}^{2} \tilde{q}_{1 \mathrm{res}}{ }^{-1} \cos ^{2} \theta+\tilde{q}_{\mathrm{W}}(1+t)}{\tilde{\varepsilon}_{2}+\tilde{q}_{2 \mathrm{res}}} .
$$

From the substitution of (24) and (16) into (21) and after some manipulations, we obtain

$$
\begin{aligned}
& \tilde{q}_{\text {leff }}^{\text {res }}= \\
& \frac{\left(\tilde{\varepsilon}_{2}+\tilde{q}_{\mathrm{W}}\right)\left[\tilde{q}_{1 \mathrm{res}} \tilde{\varepsilon}_{2}^{2}+\left(\tilde{q}_{\mathrm{w}} \tilde{q}_{2 \mathrm{res}}-1\right) \cos ^{2} \theta \tilde{\varepsilon}_{2}+\tilde{q}_{1 \mathrm{res}}-\left(\tilde{q}_{2 \mathrm{res}}+\tilde{q}_{\mathrm{W}}\right) \cos ^{2} \theta\right]}{\left(\tilde{\varepsilon}_{2}+\tilde{q}_{2 \mathrm{res}}\right)\left[\sin ^{2} \theta\left(\tilde{\varepsilon}_{2}^{2}+1\right)+\cos ^{2} \theta\left(\tilde{\varepsilon}_{2}+\tilde{q}_{\mathrm{W}}\right)^{2}\right]}
\end{aligned}
$$

where $\tilde{q}_{\text {lreseff }}$ is denoted as $\tilde{q}_{\text {leff }}^{\text {res }}$ for compact visibility. Placing $\theta=0$ into $(25)$ reduces $\tilde{q}_{\text {leff }}^{\text {res }}$ to $\tilde{q}_{\text {leff }}$ in the 3 channel QDT :

$$
\begin{aligned}
& \tilde{q}_{\text {leff }}^{\text {res }} \underset{\theta=0}{\longrightarrow} \\
& \frac{\left[\tilde{q}_{1 \mathrm{res}} \tilde{\varepsilon}_{2}^{2}+\left(\tilde{q}_{\mathrm{W}} \tilde{q}_{2 \mathrm{res}}-1\right) \tilde{\varepsilon}_{2}+\tilde{q}_{1 \mathrm{res}}-\left(\tilde{q}_{2 \mathrm{res}}+\tilde{q}_{\mathrm{W}}\right)\right]}{\left(\tilde{\varepsilon}_{2}+\tilde{q}_{2 \mathrm{res}}\right)\left(\tilde{\varepsilon}_{2}+\tilde{q}_{\mathrm{W}}\right)} \\
& \quad=\tilde{q}_{\text {leff }}(2 \mathrm{c} 1 \mathrm{o}) .
\end{aligned}
$$

It was already shown that $\tilde{q}_{\text {1res }}$ and $\tilde{q}_{\text {2res }}$ have the same form as $\tilde{q}_{1}$ and $\tilde{q}_{2}$ in the 3 channel QDT, respectively, when $\theta=0$ and $\tilde{d}_{\text {res }}$ is regarded as $\tilde{D}_{3}$. This together with (26) means that the gross features of the autoionization spectra depend only on the scalar product of $\tilde{\xi}_{1} \cdot \tilde{\xi}_{2}$. If additional open channels cannot alter the value of the scalar product, their presence cannot be felt in the spectra.

A previous study ${ }^{9}$ considered the case of the null transition $\tilde{\mathbf{D}}^{\mathrm{o}}=0$ to the open channels frequently encountered in the spectra. In this case, $\tilde{q}_{\text {ires }} \rightarrow \infty(i=1,2)$ but its product with $d_{\text {res }}$ is finite. By taking the limit of $\tilde{q}_{\text {ires }} \rightarrow \infty(i=1,2)$, multiplying 
and dividing both the numerator and denominator of (25) by $\tilde{d}_{\text {res }}$ and $\tilde{d}_{\text {res }} \tilde{q}_{\text {1res }}$, respectively, we obtain

$$
\tilde{q}_{1 \text { eff }}^{\text {res }}=\frac{r \cos \theta\left(\tilde{\varepsilon}_{2}+\tilde{q}_{\mathrm{W}}\right)\left[\tilde{\varepsilon}_{2}^{2}+\tilde{q}_{\mathrm{W}} t \tilde{\varepsilon}_{2}+1-t\right]}{\sin ^{2} \theta\left(\tilde{\varepsilon}_{2}^{2}+1\right)+\cos ^{2} \theta\left(\tilde{\varepsilon}_{2}+\tilde{q}_{\mathrm{W}}\right)^{2}}
$$

which is the improved version of Eq. (41) of Ref. [9]. Note that there is no simple pole at $\tilde{\varepsilon}_{2}=-\tilde{q}_{2 \text { res }}$ in this case, which is in contrast to (25) and (26). Eq. (27) is also reduced to $\tilde{q}_{\text {leff }}$ for the systems involving 2 closed and 1 open channel when $\theta=0$.

Systems are sometimes encountered where the null transition dipole moments $\tilde{\mathbf{D}}^{\circ}$ to the open space and there are null indirect couplings between closed channels so that $\theta$ is $\pi / 2$. In this case, $t$ is zero but $\tilde{q}_{\mathrm{w}} t$ is not zero and becomes $\tilde{q}_{\mathrm{w} 0} / r$, where $\tilde{q}_{\mathrm{w} 0}=\tilde{K}_{12} /\left(\tilde{\xi}_{1} \tilde{\xi}_{2}\right)$. Similarly, $\cos \theta\left(\tilde{\varepsilon}_{2}+\tilde{q}_{\mathrm{w}}\right)$ is not zero but becomes $\tilde{q}_{\text {wo }}$. Taking these into account, (27) can be reduced further to

$$
\tilde{q}_{\text {leff }}^{\text {res }}=\frac{r \tilde{q}_{\mathrm{w} 0}\left(\tilde{\varepsilon}_{2}^{2}+\tilde{q}_{\mathrm{w} 0} r^{-1} \tilde{\varepsilon}_{2}+1\right)}{\tilde{\varepsilon}_{2}^{2}+1+\tilde{q}_{\mathrm{w} 0}^{2}} .
$$

Note that the zero surface at $\tilde{\varepsilon}_{2}+\tilde{q}_{\mathrm{W}}=0$ in (27) disappeared. The quadratic form of the numerator of the right side of (28) enjoys several distinctive relationships. The unity of the constant term means that the two roots are multiplicative inverses of each other and limit the values of double roots to \pm 1 . The sum of the roots given by $-\tilde{q}_{\mathrm{w} 0} r^{-1}$ is equal to $-\tilde{K}_{12} \tilde{D}_{2} /\left(\tilde{W}_{2} \tilde{D}_{1}\right)$. This suggests that if $\tilde{W}_{2}$ approaches zero, one of the roots approaches infinity whereas the other approaches zero, which is equivalent to the statement that one of the roots approaches the resonance point of the interloper, whereas the other root becomes off-resonant. This means that $q$ reversal occurs at the resonance peak of an interloper and another $q$ reversal takes place around the minimum of the cross sections. This topic was discussed extensively from the viewpoint of Feshbach type's theory of resonance in Ref. [5]. The sign of the double root was determined by the sign of $\tilde{K}_{12} \tilde{D}_{2} / \tilde{D}_{1}$, suggesting that the relative sign of $\tilde{D}_{2} / \tilde{D}_{1}$ and the sign of $\tilde{K}_{12}$ are involved in the interference pattern that determines the spectral shape. Therefore, this study shows the parameters involved in the spectral shape and how they affect the shape.

Another frequently observed $q$ reversal pattern, where the autoionizing satellite peaks of the perturbed series 1 lean toward the central peak due to the interloper, is related to the sign of $r \tilde{q}_{\mathrm{w} 0}$ of (28). The frequently observed $q$ reversal pattern of the satellite peaks leaning toward the central peak corresponds to the negative sign of $r \tilde{q}_{\mathrm{w} 0}$. If the sign is positive, the $q$ reversal pattern would be such that the satellite peaks of perturbed

Table 1. The terms whose signs determine the $q$ reversal patterns.

\begin{tabular}{ll}
\hline \multicolumn{1}{c}{ Case } & \multicolumn{1}{c}{ Terms } \\
\hline General & $\tilde{q}_{\text {1res }}$ \\
$\tilde{\mathbf{D}}^{\mathrm{o}}=0$ & $\mathrm{R} \cos \theta$ \\
$\tilde{\mathbf{D}}^{\mathrm{o}}=0, \theta=\pi / 2$ & $r \tilde{q}_{\mathrm{w} 0}$ \\
$\theta=\pi / 2$ & $\tilde{q}_{\text {1res } 0} \tilde{q}_{\mathrm{w} 0}$
\end{tabular}

series 1 lean outward from the central peak. This relationship of the sign of the coefficient of the highest order term of the numerator for the formulae of $\tilde{q}_{\text {leff }}^{\text {res }}$ to the $q$ reversal pattern is general and applies to all cases. Table 1 lists the coefficient of the highest order terms of the numerator of $\tilde{q}_{\text {leff }}^{\text {res }}$ that determine the $q$ reversal patterns in various cases.

The shape of photoionization cross section (6) is determined by $\tilde{q}_{\text {leff }}^{\text {res }}$, which in turn is determined only by $\tilde{q}_{\mathrm{w}}, \tilde{q}_{\text {1res }}, \tilde{q}_{\text {2res }}$ and $\theta\left(I_{2}\right.$ and $\mu_{2}$ are also needed for $\left.\tilde{\varepsilon}_{2}\right)$. All the parameters $\tilde{D}_{1}, \tilde{D}_{2}$, $\tilde{d}_{\text {res }}, \tilde{\xi}_{1}, \tilde{\xi}_{2}, \theta$ and $\tilde{K}_{12}$ must have been considered instead of mere $\tilde{q}_{\mathrm{W}}, \tilde{q}_{\text {1res }}, \tilde{q}_{\text {2res }}$ and $\theta$ if the analytical form of $\tilde{q}_{\text {leff }}^{\text {res }}$ is not known. Although the number of parameters are reduced considerably, the functional behavior of zero surfaces of $\tilde{q}_{\text {leff }}^{\text {res }}\left(\tilde{\varepsilon}_{2}\right)=0$ for $q$ reversal analysis is still determined by as many as 5 parameters, $\tilde{q}_{\mathrm{w}}, \tilde{q}_{\text {1res }}, \tilde{q}_{\text {2res }}, \theta$ and $\tilde{\varepsilon}_{2}$, and cannot be visualized in 3 dimensional space. On the other hand, if the transition dipole moments to the open channels are zero, only 3 parameters are needed for the zero surfaces of $\tilde{q}_{1 \text { eff }}^{\text {res }}\left(\tilde{\varepsilon}_{2}\right)$ and can be visualized, as was done in Ref. [9].

Both (25) and (27) show that when $\theta=0,\left(\tilde{\varepsilon}_{2}+\tilde{q}_{\mathrm{W}}\right)^{2}$, which is the second order pole at the denominator in (27) cancels $\tilde{\varepsilon}_{2}+\tilde{q}_{\mathrm{W}}$ out in the numerator and becomes a simple pole. In other words, one of the zero surfaces, $\tilde{\varepsilon}_{2}+\tilde{q}_{\mathrm{W}}=0$, is transformed to a simple pole. This change leaves the $q$ reversal patterns unaltered but huge intensity borrowing appears around the simple pole as a new phenomenon for systems involving only 1 open channel. In contrast, a huge peak due to intensity borrowing disappears when additional open channels are included and the autoionization spectra vary more smoothly.

The role of open channels in resonance phenomena and its limiting cases. If more than 1 open channel is involved in autoionization spectra, only one eigenchannel acts as a resonance eigenchannel and the others only contribute to the background spectra. This means that the number of effective continuum remains unaltered when the number of open channels increases. An increase in the number of open channels increases the dimensionality of the space spanned by the open channels and is accompanied by an increase in the indirect coupling paths that are equal to the number of open channels, making it easy to interfere destructively. In this space, let us represent the eigenchannel corresponding to the resonance part by the vector $\mathbf{Z}_{\text {res }}^{\mathrm{o}}$, coupling of the closed channels with open ones by vectors $\xi_{1}$ and $\tilde{\xi}_{2}$ and the transition dipole moments to open channels by

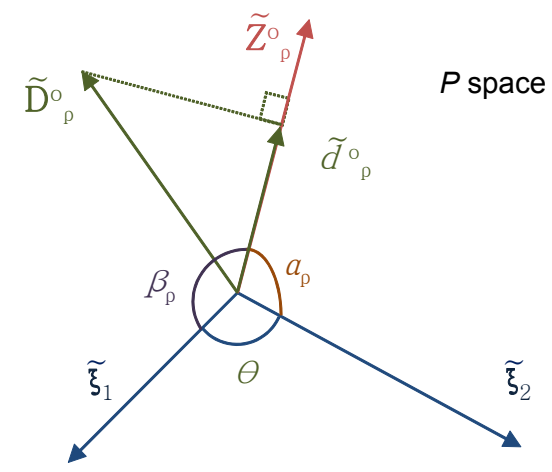

Figure 1. Various vectors and direction cosines in open channel space $P$. 
the vector $\tilde{\mathbf{D}}^{\mathrm{o}}$ as shown in Fig. 1. The degree of destructive interference in indirect coupling is represented in this space by the angle $\theta$ that two coupling vectors $\tilde{\xi}_{1}$ and $\tilde{\xi}_{2}$ make. The magnitude of the line profile indices $\tilde{q}_{1 \text { res }}$ and $\tilde{q}_{\text {2res }}$ are proportional to

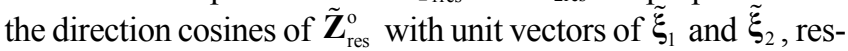
pectively. The projection of the transition dipole moment vector $\tilde{\mathbf{D}}^{\circ}$ to $\widetilde{\mathbf{Z}}_{\text {res }}^{\mathrm{o}}$ determines its actual contribution to the cross sections.

As the indirect coupling $\left(\tilde{\xi}_{1}\right)_{i}\left(\tilde{\xi}_{2}\right)_{i}=\tilde{K}_{1 i} \tilde{K}_{2 i}$ via the open channel $i$ comprises one component of the scalar product of two coupling vectors $\tilde{\xi}_{1}$ and $\tilde{\xi}_{2}$, destructive interference can be visualized as the scalar product of two coupling vectors $\tilde{\xi}_{1}$ and $\tilde{\xi}_{2}$ in open channel space $P$. Complete destructive interference corresponding to the null indirect coupling occurs when two vectors are perpendicular to each other, or when $\theta=\pi / 2$. The maximum contribution of indirect coupling occurs when two vectors are parallel to each other so that $\theta=0$ or $\pi$. In this case, all the behaviors of the autoionization spectra of the resonant eigenchannels are the same as those of the 3 channel QDT, except at the discontinuity points, as already shown in (26).

For the zero indirect coupling case corresponding to $\tilde{\xi}_{1} \cdot \tilde{\xi}_{2}=$ $0, \tilde{q}_{\mathrm{w}}$ defined by (18) approaches infinity while $\tilde{q}_{\mathrm{w}} \cos \theta$ remains finite and equal to $-\tilde{K}_{12} / \tilde{\xi}_{1} \tilde{\xi}_{2}$. Let us denote this finite product as $\tilde{q}_{\mathrm{w} 0}$. Numerous spectra belonging to this null indirect coupling case have been observed, particularly by Hogervorst's group. ${ }^{16-19}$ One of their spectra will be considered in the next section. They applied extensively the concept of assigning one effective continuum to one closed channel proposed by Cooke and Cromer. ${ }^{4}$ They also introduced the simplification to the proposal and limited the nonzero values of $\tilde{K}_{i m}^{\mathrm{co}}$ to the ones with indices satisfying $m=n_{\mathrm{c}}+i$ ( $n_{\mathrm{c}}$ : the number of closed channels). This limitation yields null $\tilde{\xi}_{i} \cdot \tilde{\xi}_{j}$ for different closed channels $i$ and $j$.

Reduced widths and energy for this null indirect coupling case are simplified as follows:

$$
\begin{aligned}
& \tilde{W}_{\text {leff }}=\tilde{W}_{1}\left(1+\frac{\tilde{q}_{\mathrm{w} 0}^{2}}{\tilde{\varepsilon}_{2}^{2}+1}\right) \geq \tilde{W}_{1} \\
& \tilde{\varepsilon}_{\text {leff }}=\tilde{\varepsilon}_{1}-\frac{\tilde{\varepsilon}_{2}}{\tilde{\varepsilon}_{2}^{2}+1} \tilde{q}_{\mathrm{W} 0}^{2} .
\end{aligned}
$$

Since there is only one path for direct coupling $\tilde{K}_{12}$, no destructive interference takes place and $\tilde{W}_{\text {leff }}$ is always $\geq \tilde{W}_{1}$. Destructive interference between direct couplings might have been possible if there were more than 2 closed channels. Eq. (29) shows that $\tilde{W}_{\text {leff }}$ and $\tilde{\varepsilon}_{\text {leff }}$ become $\tilde{W}_{1}$ and $\tilde{\varepsilon}_{1}$, respectively, if $\tilde{K}_{12}$ is zero, meaning that the resonances are isolated as a matter of course because in this case, the indirect coupling of closed channels and the direct coupling are zero. If the direct coupling $\tilde{K}_{12}$ is turned on between closed channels 1 and 2 in the absence of an indirect one, the perturbations of the interloper begin to affect the spectral width $\tilde{W}_{1}$, reduce the energy $\tilde{\varepsilon}_{1}$ of Rydberg series 1 and contribute to additional terms proportional to $\tilde{K}_{12}^{2}$. Strictly, it is not $\tilde{K}_{12}^{2}$ but $\tilde{q}_{\text {w0 }}^{2}$ that is equal to $\tilde{K}_{12}^{2} /\left(\tilde{\xi}_{1}^{2} \tilde{\xi}_{2}^{2}\right)$. This highlights the importance of the vector lengths of the coupling vectors $\tilde{\xi}_{1}$ and $\tilde{\xi}_{2}$ besides the angle between them. Although for the null indirect coupling case, the scalar product $\tilde{\xi}_{1} \cdot \tilde{\xi}_{2}$ representing the indirect coupling in space $P$ is zero, and there is only direct coupling between the closed channels which has no representation in space $P$, the additional open channels may still affect the autoionization dynamics. The above result shows that this is indeed the case, and the open channels affect the dynamics through the vector lengths of the coupling vectors. The effect of direct coupling on the autoionization dynamics is diminished as the vector lengths of coupling vectors increase. This suggests that direct coupling is not fully compatible with the autoionization dynamics, and this incompatibility in conjunction with the uncertainty principle of quantum mechanics causes depolarizing quantum averaging. With this interpretation, the meaning of $\tilde{q}_{\text {wo }}$ can be given as the depolarized strength of the direct coupling.

Let us examine the behavior of $\tilde{W}_{\text {leff }}$ of (29), which is the measure of the autoionization rate of a perturbed Rydberg series 1 and whose energy variation is caused by coupling from the interloper. The maximum of $\tilde{W}_{\text {leff }}$ occurs at the resonance point $\tilde{\varepsilon}_{2}=0$ of an interloper series and is given by $\tilde{W}_{\text {leff }}^{\max }=\tilde{W}_{1}+$ $\tilde{K}_{12}^{2} / \tilde{W}_{2}$. This indicates that the maximum autoionization rate of the perturbed series 1 increases with increasing direct coupling strength $\tilde{K}_{12}$ with an interloper. The increase in the maximum rate of series 1 with increasing $\tilde{K}_{12}$ may be due to either the leakage of series 2 into series 1 or the leakage of series 1 into series 2 . Interestingly, the maximum rate of series 1 decreases as the rate of series 2 gauged by $\tilde{W}_{2}$ increases, indicating that the autoionization of series 2 blocks the autoionization of series 1 . This also suggests that an increase in the maximum rate of series 1 with increasing $\tilde{K}_{12}$ is caused mainly by the leakage of series 2 into series 1 . Note that the maximum rate approaches infinity as the autoionizing rate of series 2 measured by $\tilde{W}_{2}$ approaches zero. However, caution should be taken when making an interpretation. The range affected by the increase in the maximum rate is given by $\tilde{W}_{2}$. Therefore, although the maximum rate of series 1 approaches infinity, its affected range also approaches zero, meaning that the infinite rate is seldom realized in the spectra.

Consider the line shape parameter $\tilde{q}_{\text {leff }}^{\text {res }}$ when $\theta=\pi / 2$. Its unperturbed version $\tilde{q}_{1 \text { res }}$ is zero from (23). Its value comes purely from the perturbation by direct coupling and is proportional to $\tilde{q}_{\mathrm{w} 0}^{2}$ :

$$
\tilde{q}_{\text {leff }}^{\text {res }}=\frac{\tilde{W}_{1}}{\tilde{W}_{\text {leff }}}\left(\frac{\tilde{\varepsilon}_{2}}{\tilde{\varepsilon}_{2}^{2}+1}-\frac{1-\tilde{q}_{1 \mathrm{res} 0} / \tilde{q}_{\mathrm{w} 0}}{\tilde{\varepsilon}_{2}+\tilde{q}_{2 \mathrm{res}}}\right) \tilde{q}_{\mathrm{w} 0}^{2},
$$

where $\tilde{q}_{1 \text { res } 0}$ denotes $\tilde{q}_{1 \text { res }} \cos ^{-1} \theta$. For only direct coupling, $\tilde{W}_{1} /$ $\tilde{W}_{\text {leff }}$ is $\leq 1$ meaning that the leakage due to the direct coupling reduces the magnitude of $\tilde{q}_{\text {leff }}^{\text {res }}$ and the overall shapes of the autoionization spectra become closer to the interloper one. Eq. (30) becomes (28) when $\tilde{\mathbf{D}}^{\circ}=$ zero, confirming the validity of the derivation.

Thus far, the behavior of the dynamic parameters for the limiting cases was examined: either $\theta=0, \pi$ or $\theta=\pi / 2$. For the cases lying in between, the scaled spectral width (16) is given by the sum of two incoherent terms with the weights given by 
$\cos ^{2} \theta$ and $\sin ^{2} \theta$. One term corresponds to the limiting case of $\theta=0$ or $\pi$ and accounts for the change in width due to interference between direct and indirect couplings with an interloper represented by their ratio, $-\tilde{q}_{\mathrm{W}}=\tilde{K}_{12} / \tilde{\xi}_{1} \cdot \tilde{\xi}_{2}$. The other term is the unperturbed case and stands for the unperturbed contribution from series 1 . Note that the $\theta=\pi / 2$ case contains contributions from both terms, as shown in (29).

Since the pole and zero surface structures of $\tilde{q}_{\text {leff }}^{\text {res }}$ greatly affect the autoionization spectra, let us consider the effect of addition open channels on the pole and zero surface structures of $\tilde{q}_{\text {leff }}^{\text {res }}$. There is one more zero surface of $\tilde{q}_{\text {leff }}^{\text {res }}$ when more than 1 open channel is involved. This zero surface becomes a simple pole if $\theta=0$ so that two resonances are completely superimposable:

$$
\begin{aligned}
& \tilde{q}_{\text {leff }}^{\text {res }}= \\
& \frac{\left(\tilde{\varepsilon}_{2}+\tilde{q}_{\mathrm{W}}\right)\left[\tilde{q}_{1} \tilde{\varepsilon}_{2}+\left(\tilde{q}_{\mathrm{W}} \tilde{q}_{2}-1\right) \cos ^{2} \theta \tilde{\varepsilon}_{2}+\tilde{q}_{1}-\left(\tilde{q}_{2}+\tilde{q}_{\mathrm{W}}\right) \cos ^{2} \theta\right]}{\left(\tilde{\varepsilon}_{2}+\tilde{q}_{2 \mathrm{res}}\right)\left[\sin ^{2} \theta\left(\tilde{\varepsilon}_{2}^{2}+1\right)+\cos ^{2} \theta\left(\tilde{\varepsilon}_{2}+\tilde{q}_{\mathrm{W}}\right)^{2}\right]} \\
& \underset{\theta=0}{\longrightarrow} \frac{\left[\tilde{q}_{1} \tilde{\varepsilon}_{2}+\left(\tilde{q}_{\mathrm{W}} \tilde{q}_{2}-1\right) \tilde{\varepsilon}_{2}+\tilde{q}_{1}-\left(\tilde{q}_{2}+\tilde{q}_{\mathrm{W}}\right)\right]}{\left(\tilde{\varepsilon}_{2}+\tilde{q}_{2 \mathrm{res}}\right)\left(\tilde{\varepsilon}_{2}+\tilde{q}_{\mathrm{W}}\right)}
\end{aligned}
$$

Although there is no difference in the fact that a line profile index changes sign at $\tilde{q}_{\mathrm{W}}$, the nature of the point of $\tilde{\varepsilon}_{2}=-\tilde{q}_{\mathrm{W}}$ is completely different.

Application to Ba $6 p_{1 / 2} n p 3 / 2 J=1$ autoionizing Rydberg series with $6 p_{3 / 2} n f$ series as an interloper. Let us apply the theory to the narrow $6 \mathrm{p}_{1 / 2,3 / 2} n \mathrm{p} J=1$ autoionizing Rydberg series in barium perturbed by the $6 p_{3 / 2} n f$ states obtained in Ref. [19]. They used $5 \mathrm{~d}_{3 / 2} 6 \mathrm{p}_{3 / 2}{ }^{3} \mathrm{P}_{0}$ as an intermediate level to excite the $6 \mathrm{p} n l J=1$ autoionizing Rydberg series. For $J=1$, there are four $6 \mathrm{p} n \mathrm{p}$ series, two converging to the $6 \mathrm{p}_{1 / 2}$ limit and two to the $6 \mathrm{p}_{3 / 2}$ limit. In addition, one rapidly autoionizing $6 \mathrm{p}_{3 / 2} n \mathrm{f}_{5 / 2}$ series occurs. Two series converging to $6 \mathrm{p}_{1 / 2} n \mathrm{p}_{1 / 2}$ and $6 \mathrm{p}_{1 / 2} n \mathrm{p}_{3 / 2}$ are not coupled strongly and series $6 \mathrm{p}_{1 / 2} n \mathrm{p}_{1 / 2}$ may be omitted without a noticeable change in the major nature of the spectra belonging to the $6 \mathrm{p}_{1 / 2} n \mathrm{p}_{3 / 2}$ series for the test of the current theory. Using this simplification, their 7 channel phase-shifted MQDT calculation, which satisfactorily reproduces the observed spectra, can be reduced to the one involving 4 channels: $6 \mathrm{p}_{1 / 2} n \mathrm{p}_{3 / 2}, 6 \mathrm{p}_{3 / 2} n f$ $5 / 2$ and two open channels.

In the spectra, the $6 \mathrm{p}_{3 / 2} n \mathrm{f}_{5 / 2}$ series acts as an interloper to the autoionizing Rydberg series $6 p_{1 / 2} n p_{3 / 2}$. The magnitudes of channel coupling with the closed channels are the parameters to be inputted for open channels. In addition to those, the other nature of the open channels is not required for the MQDT calculation. The present theory clarifies this point and shows that open channels enter the formalism only in the form of $\tilde{\xi}_{i}$ and $\theta$. For concreteness, the experimental identifications with $6 \mathrm{~s} \varepsilon l$ for the decay of the $6 \mathrm{p} n \mathrm{p}$ series and $5 \mathrm{~d} \varepsilon l^{\prime}$ for the decay of the $6 \mathrm{p}_{3 / 2} n \mathrm{f}$ series, which are compatible with the selection rule, $\Delta l= \pm 1$, may be specified. The number of open channels does not affect the fit provided greater than one because their contributions might be regarded in an average sense as a constant function of energy, as in the configuration mixing theory of Fano. $^{20}$ They only affect the fit through the angle $\theta$ between the
Table 2. MQDT parameters for the $6 \mathrm{p}_{1 / 2} n \mathrm{p}_{3 / 2}$ and $6 \mathrm{p}_{3 / 2} n \mathrm{f}_{5 / 2} J=1$ series of $\mathrm{Ba}$ in 4 channel analysis.

\begin{tabular}{ccccc}
\hline & $\begin{array}{c}i=1 \\
6 \mathrm{p}_{1 / 2} n \mathrm{p}_{3 / 2}\end{array}$ & $\begin{array}{c}i=2 \\
6 \mathrm{p}_{3 / 2} n \mathrm{f}_{5 / 2}\end{array}$ & $\begin{array}{c}i=3 \\
6 \mathrm{~s} \varepsilon l\end{array}$ & $\begin{array}{c}i=4 \\
5 \mathrm{~d} \varepsilon l^{\prime}\end{array}$ \\
\hline $\mathrm{I}_{i}\left(\mathrm{~cm}^{-1}\right)$ & 62296.46 & 63987.32 & 42034.90 & 46908.75 \\
$\mu_{i}$ & 3.781 & 0.08 & & \\
$\tilde{D}_{i}$ & -0.5 & 5 & 0 & 0 \\
& \multicolumn{4}{c}{ Nonzero $\tilde{K}$ matrix elements } \\
& $\tilde{K}_{12}=-0.11, \tilde{K}_{13}=-0.08, \tilde{K}_{24}=0.58$ & \\
\hline
\end{tabular}
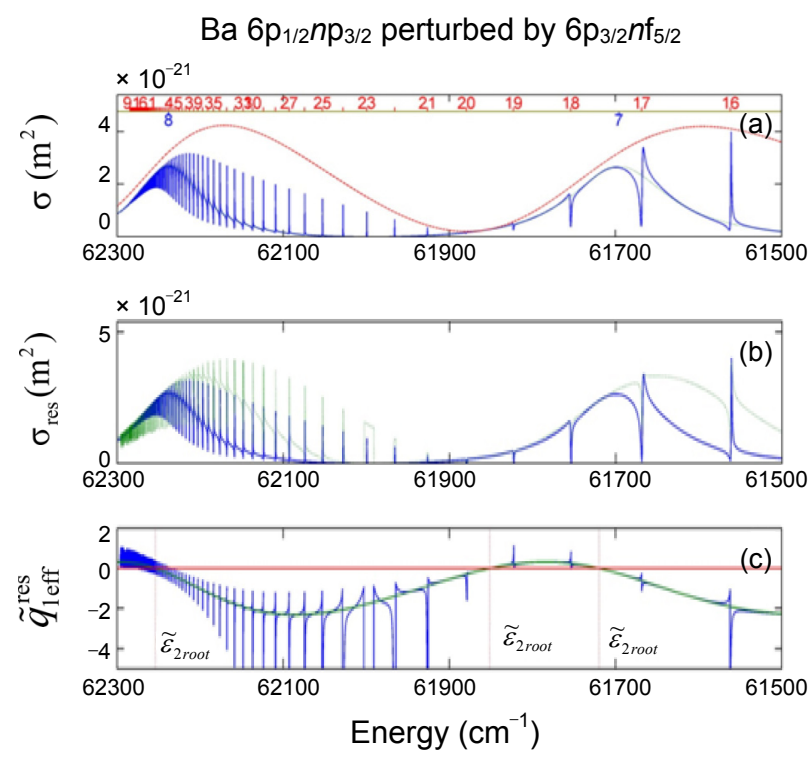

Figure 2. (a) Spectrum resulting from 4-channel QDT with the parameters in Table 2. The cross section $\sigma$ is drawn with a solid line, the envelope with a dotted line and $\sigma_{\max }$ with a dashed line. Effective quantum numbers are shown in the $\sigma$ spectrum. The upper integer values are $n_{1}{ }^{*}$ for the perturbed Rydberg series and the lower one is $n_{2}{ }^{*}$ for the interloper. The inability of $\sigma_{\max }$ to follow the peak maxima around $n_{2}{ }^{*}=8$ is due to instrumental smoothening. (b) The spectrum corresponding to the resonant eigenchannel component. (c) The line profile spectra of the perturbed series 1 drawn with Eqs. (21) and (28).

coupling vectors $\tilde{\xi}_{1}$ and $\tilde{\xi}_{2}$.

The relevant parameters in Table 1 were copied from Ref. [19] for self containment. Note that in this system, the interaction of closed channel 1 with open channels is non-zero only with channel 3, i.e., $\tilde{\xi}_{1}=[-0.080]$ and closed channel 2 only with channel 4 , i.e., $\tilde{\xi}_{2}=\left[\begin{array}{ll}0 & 0.58\end{array}\right]$ so that $\tilde{\xi}_{1} \cdot \tilde{\xi}_{2}=0$, or two coupling vectors are perpendicular to each other so that $\theta=\pi / 2$. Each closed channel couples with its own effective continuum, resulting in null indirect coupling. Although closed channels do not interact indirectly via open channels, they can interact directly with each other but with the strength $\tilde{K}_{12}$ reduced by $\tilde{\xi}_{1} \tilde{\xi}_{2}$.

Fig. 2(a) shows the spectrum calculated using the 4 channel QDT with the parameters in Table 2. Fig. 2(b) shows the spectrum of the resonance part and confirms the assertion that it comprises most of the total cross sections. Fig. 2(c) shows the spectrum of the line profile index $\tilde{q}_{\text {leff }}^{\text {res }}$ of the perturbed autoionizing Rydberg series 1 . The straddling intervening re- 
Ba $6 p_{1 / 2} n p_{3 / 2}$ perturbed by $6 p_{3 / 2} n f_{5 / 2}$ (3 channels)
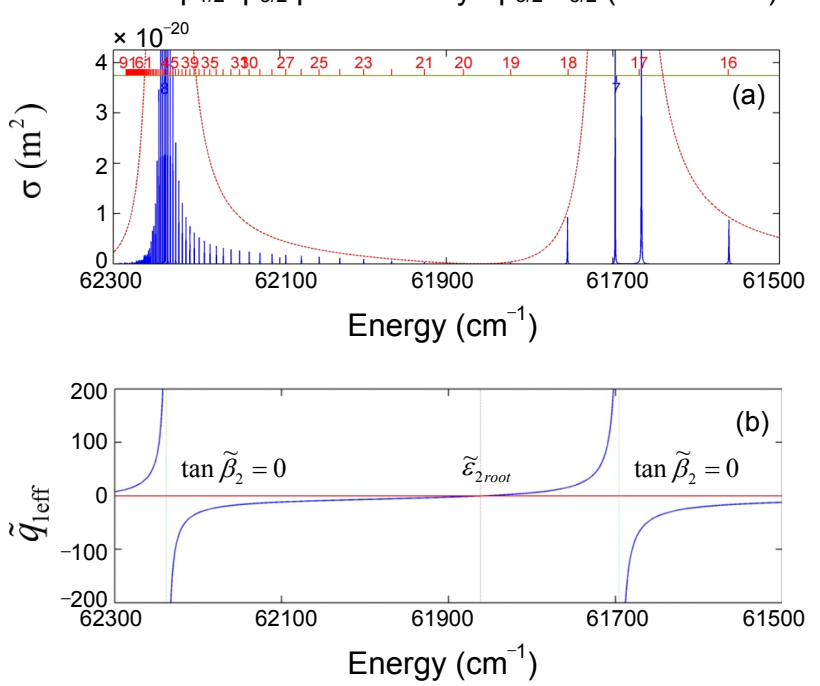

Figure 3. Photoionization cross section and $\tilde{q}_{\text {leff }}$ spectra calculated using the 3 channel QDT with the same parameters as those for Fig. 2 except that $\tilde{K}_{24}=0$. The line profile spectrum is drawn with (C2).

sonance peak corresponding to $6 \mathrm{p}_{3 / 2} 7 \mathrm{f}_{5 / 2}$ around which the perturbed autoionizing Rydberg series 1 change sign in $q$ can be identified in Fig. 2(a). The closeness of the $q$ value to zero in a wide range of energies around the straddling peak makes the spectra follow the spectra of the interloper more smoothly. If $\tilde{K}_{24}$ is zero, the system is reduced to a 3 channel one involving 1 open channel. Fig. 3 shows the spectrum calculated using the 3 channel QDT, i.e., with the same parameter as those for Fig. 2 except that $\tilde{K}_{24}=0$. Note the completely different appearance and nature of the two spectra. It is difficult to identify the separate resonance peaks of the interloper at $n_{2}^{*}=7$ in Fig. 2(a). Instead, it acts as a structured background to the perturbed series 1 . On the other hand, it is clearly discernable as a separate peak in Fig. 3(a). Here, caution should be taken when interpreting the narrow peaks of series 1 in Fig. 2(a) as the window-like resonances due to the overlapping narrowing observed by Mies. ${ }^{14}$ In that case, the peaks should lie in the middle of the original peaks of series 1, as clearly shown by Giusti-Suzor and Fano $^{3}$ in contrast to the spectrum in Fig. 2(a). Fig. 3(a) shows huge intensity borrowing around the poles, which is the newly emerged feature by the change from 4 to 3 channels.

Within the restriction of no transition to the open channels and null indirect coupling, the spectra can still show a different type as $\tilde{K}_{24}$ varies. According to Fig. 4 , the roots of $\tilde{\tilde{q}}_{\text {leff }}^{\text {res }}\left(\tilde{\varepsilon}_{2}\right)=0$ disappear after merging into one with value unity at $\tilde{K}_{24}$ given by $\sqrt{\tilde{K}_{12} \tilde{D}_{2} /\left(2 \tilde{D}_{1}\right)}$, whose value is 0.74 . (If the sign of $\tilde{K}_{12} \tilde{D}_{2} / \tilde{D}_{1}$ is negative, $\tilde{K}_{24}$ would be given by $\sqrt{-\tilde{K}_{12} \tilde{D}_{2} /\left(2 \tilde{D}_{1}\right)}$ with the double root corresponding to the value of -1 .) The straddling intervening $q$ reversal around the central peak due to the inter loper would then disappear, as shown in Fig. 5, whose spectrum corresponds to $\tilde{K}_{24}=0.9$. This application shows that the explicit formula for $\tilde{q}_{\text {leff }}^{\text {res }}$ obtained in the previous section can be used as a powerful tool for determining the entire range of possible spectral patterns.

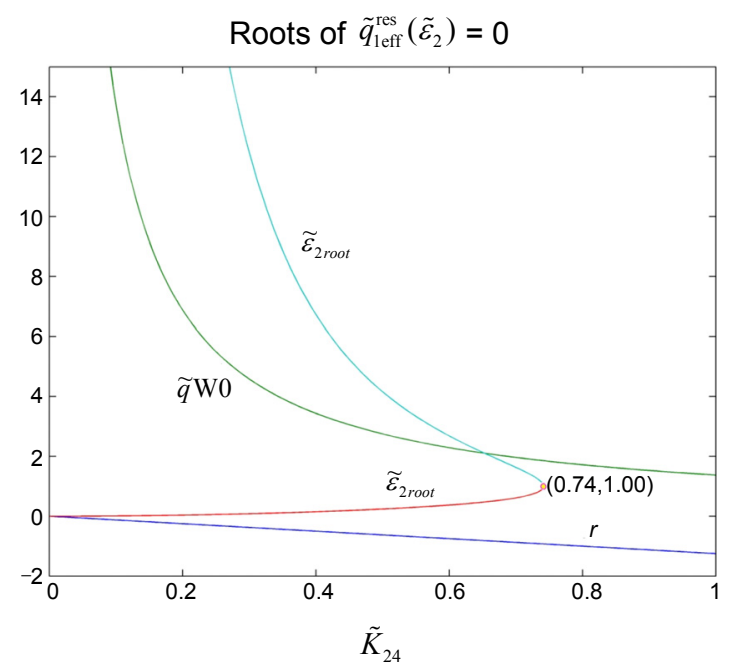

Figure 4. Behavior of the roots of $\tilde{q}_{\text {leff }}^{\text {res }}\left(\tilde{\varepsilon}_{2}\right)=0$ and relevant parameters as a functions of $\tilde{K}_{24}$.
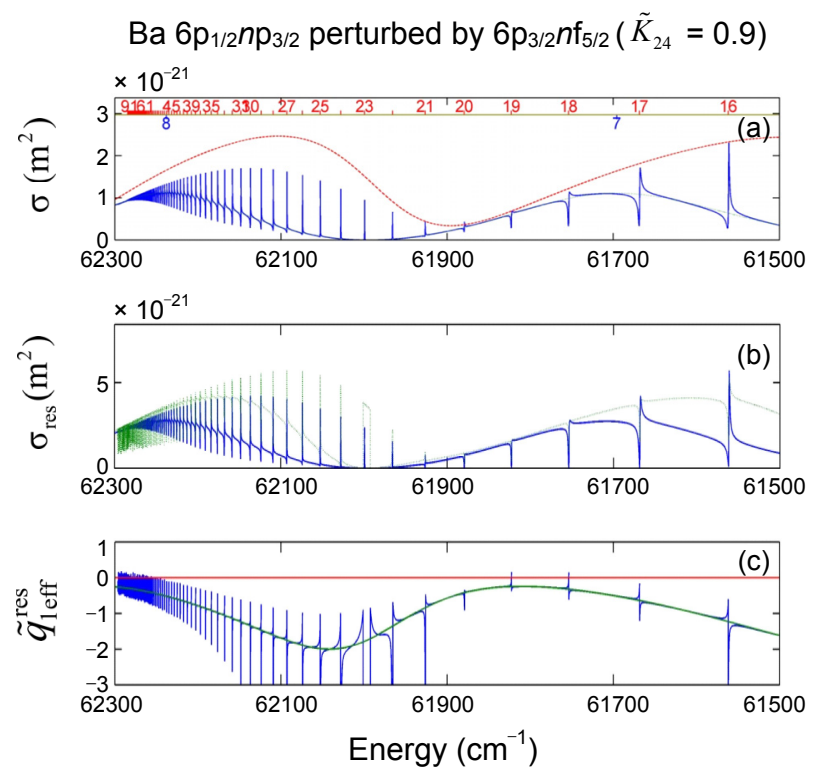

Figure 5. The spectrum resulting from 4-channel QDT with the same parameters as those of Fig. 2 except that $\tilde{K}_{24}=0.9$.

\section{Result and Discussion}

A Rydberg series perturbed by an interloper is one of the important systems showing complex resonance phenomena due to the overlapping resonance. Ueda's formulation ${ }^{7}$ or a similar one by Cooke and Cromer ${ }^{4}$ provides the decomposition of the autoionization spectra into those of the interloper and the perturbed autoionizing Rydberg series by it. Although the decomposition provides an analysis tool for examining the mutual effects between the interloper and perturbed autoionizing series, each decomposed part was found to be prone to the contamination with poles. The poles are not present in the undecomposed form. They only emerge in the decomposed form and are considered intrinsic in the decomposed formulae and cannot 
be removed. Indeed, the observed spectra were influenced greatly by the pole structures, whose effect was toned down by the contribution from concurrent incoherent processes. With the explicit factoring of the pole structure sometimes with the help of the decomposition into the partial fractions, the previous study turned the problematic poles into powerful tools for examining autoionization spectra. ${ }^{8}$

When additional open channels are involved, further contamination with kinks and poles occurs because of the connection nature of eigenchannels into the resonant and non-resonant ones proposed by Cohen. ${ }^{11}$ His proposal to construct resonant dominant and non-resonant dominant eigenchannels was the critical step to the extension of the Ueda type of decomposition to the systems involving more than 1 open channel, which allowed us to apply Ueda's formulation to the resonant eigenchannel and treat the contributions of remaining non-resonant part as a background. However, such attempts were hampered by the difficulty to obtain formulae with the structures of pole explicitly exposed except for some particular cases, and only partial success was reported. ${ }^{9}$

This study examined this problem again. The removal of kinks was achieved using the solutions at the most remote points from the kinks. The solutions corresponded to the effective continuum $^{4,14}$ or Fano's a state ${ }^{15}$ for the autoionizing Rydberg series 2. In physical terms, such removal of kinks helps replace the perturbed dynamic constants pertaining to the open channels with no structure in the autoionization processes for Rydberg series 1 with a structured background that is provided by the envelope of the interloper. For example, the line profile index $\tilde{q}_{\text {leff }}^{\text {res }}$ is no longer constant. Its energy variation follows the structured background provided by the interloper.

The explicit formulae for the perturbed autoionizing Rydberg series thus obtained for the general cases show the structures of troublesome poles and extremely valuable zero surfaces of the spectral shape parameter $q$. Without knowing such structures, the behavior of the dynamic parameters would not be controlled easily because of their peculiar behavior due to the singularity. The explicit formulae helped identify the parameters responsible for the observed phenomena, such as for the frequently observed $q$ reversal pattern, where the autoionizing satellite peaks of the perturbed series 1 lean toward the central peak due to the interloper. The table was given to show the terms that are responsible for the observed phenomena, in particular for the $q$ reversal, which is the most important single parameter that determines the spectral line shapes of autoionization.

In order to emphasize the effect of the additional open channels on the autoionization spectra, the case of extreme deviation from the 1 open channel systems was studied in depth. Such extreme deviation corresponds to the null indirect coupling case, which occurs when the indirect coupling paths between the closed channels via the open channels in space $P$ (consisted of open channels) interfere destructively and there remains only direct coupling between the closed channels, which has no representation in space $P$. Although no coupling of the closed channels occurs in space $P$, the open channels may still affect the autoionization dynamics not by indirect coupling represented by the scalar product between the coupling vectors in space $P$ but by the vector lengths of the coupling vectors in space $P$.
The effect of direct coupling on the autoionization dynamics decreased with increasing the vector lengths of the coupling vectors in space $P$. This suggests that the direct coupling is not fully compatible with the autoionization dynamics, and this incompatibility in conjunction with the uncertainty principle of quantum mechanics causes depolarizing quantum averaging. With this interpretation, the meaning of $\tilde{q}_{\mathrm{wo}}$ was given as the depolarized strength of the direct coupling.

The present theory was applied to the narrow $6 \mathrm{p}_{1 / 2,3 / 2} n \mathrm{p} J=1$ autoionizing Rydberg series in barium perturbed by the $6 \mathrm{p}_{3 / 2} n \mathrm{f}$ states obtained by de Graaff et al., ${ }^{19}$ which is one of the series of experiments that applies the concept of assigning one effective continuum to one closed channel proposed by Cooke and Cromer. ${ }^{4}$ The experiment corresponds to the case of null indirect coupling between the closed channels in our theory. To emphasize the effect of additional open channels to autoionization spectra, the transformation from the observed autoionization spectrum into the one for the 3 channel system was performed by tending one of the coupling parameters to zero. A completely different spectrum was obtained: the resonance peaks in the 4 channel system act as a structured background to the perturbed autoionizing series 1 , whereas it is clearly discernable as a separate peak in the 3 channel system and huge intensity borrowing around the interloper resonance peak occurs. In addition to this transformation from a 4 to 3 channel system, the theory was used to identify other possible different types of spectra by varying the same coupling parameter. One of the limits of this application was that the number of closed channels needs to be reduced from 5 to 2 to apply the theory. Therefore, future work will need to extend the theory to systems with more than 2 closed channels, in particular to systems that still keep the scheme of a perturbed autoionizing series by an interloper but allow degeneracy either or both for the perturbed series and interloper.

Acknowledgments. This study was supported by the Ajou university research fellowship of 2009 under contract No. 20095142COR0101S000100.

\section{Appendix}

\section{Appendix A: Decomposition of the Resonance Phase Shifts into the Incoherent Sum.}

Let us obtain the explicit form of the resonance phase shifts $\tilde{\delta}_{\text {r }}$ which can be obtained from the phase of $\tan \tilde{\beta}+\tilde{\kappa}^{\mathrm{cc}}$. From $\tan \tilde{\beta}+\tilde{\kappa}^{\mathrm{cc}}=C \exp \left(i \tilde{\delta}_{\mathrm{r}}\right)$ and after some manipulations, the following can be obtained:

$$
C \mathrm{e}^{\mathrm{i} \tilde{\delta}_{r}}=\left(\tan \tilde{\beta}_{1}-\mathrm{i} \tilde{\xi}_{1}^{2}\right)\left(\tan \tilde{\beta}_{2}-\mathrm{i} \tilde{\xi}_{2}^{2}\right)+\left(\tilde{\xi}_{1} \cdot \tilde{\xi}_{2}+\mathrm{i} \tilde{K}_{12}\right)^{2}
$$

Note that $\tan \tilde{\beta}_{i} / \tilde{\xi}_{i}^{2}=\tan \tilde{\beta}_{i} / \tilde{W}_{i}=\tilde{\varepsilon}_{i}$. It is also equal to $-\cot \tilde{\delta}_{i}$. Eq. (A1) can then be written as follows:

$$
C \mathrm{e}^{\mathrm{i} \tilde{\delta}_{r}}=\tilde{W}_{1} \tilde{W}_{2}\left[\left(\tilde{\varepsilon}_{1}-\mathrm{i}\right)\left(\tilde{\varepsilon}_{2}-\mathrm{i}\right)+\left(1-\mathrm{i} \tilde{q}_{\mathrm{W}}\right)^{2} \cos ^{2} \theta\right]
$$

Note that Eq. (A2) is symmetric. The next crucial step is to break this symmetry in the formula to incorporate the asymmetry 
imposed by the physical situation of the rapidly varying series perturbed by a slowly varying one, the interloper, for a given energy interval. Let us call the rapidly varying series series 1 and the slowly varying one series 2 . Since series 2 varies slowly, it will act as an envelope to series 1 . This can be incorporated into (A2) by factoring $\tilde{\varepsilon}_{2}$-i out:

$$
C \mathrm{e}^{i \tilde{\delta}_{r}}=\tilde{W}_{1} \tilde{W}_{2}\left(\tilde{\varepsilon}_{2}-\mathrm{i}\right)\left[\left(\tilde{\varepsilon}_{1}-\mathrm{i}\right)+\frac{\left(1-\mathrm{i} \tilde{q}_{\mathrm{W}}\right)^{2}}{\left(\tilde{\varepsilon}_{2}-\mathrm{i}\right)} \cos ^{2} \theta\right] .
$$

(A3) can be transformed by separating the terms in the bracket on the right side of (A3) into real and imaginary parts, as follows:

$$
C \mathrm{e}^{i \tilde{\delta}_{r}}=\tilde{W}_{\text {leff }} \tilde{W}_{2}\left(\tilde{\varepsilon}_{2}-\mathrm{i}\right)\left(\tilde{\varepsilon}_{\text {leff }}-\mathrm{i}\right)
$$

where $\tilde{W}_{\text {leff }}$ and $\tilde{\varepsilon}_{\text {leff }}$ are the intrinsic spectral width and reduced energy of the perturbed series in (1) defined in (16) and (17), respectively. Substituting (15) into (A4) yields

$$
\mathrm{e}^{i \tilde{\delta}_{\mathrm{r}}}=\left[\frac{\left(\tilde{\varepsilon}_{\text {leff }}-i\right)\left(\tilde{\varepsilon}_{2}-\mathrm{i}\right)}{\left(\tilde{\varepsilon}_{\text {leff }}+i\right)\left(\tilde{\varepsilon}_{2}+\mathrm{i}\right)}\right]^{1 / 2} .
$$

Since $\exp \left(i \tilde{\delta}_{\mathrm{r} j}\right)=\left[\left(\tilde{\varepsilon}_{j}-\mathrm{i}\right) /\left(\tilde{\varepsilon}_{j}+\mathrm{i}\right)\right]^{1 / 2}$, (A5) indicates that

$$
\tilde{\delta}_{\mathrm{r}}=\tilde{\delta}_{\mathrm{rleff}}+\tilde{\delta}_{\mathrm{r} 2}
$$

with $j$ corresponding to 1 eff and 2, respectively. Eq. (A6) means that resonance scattering is decomposed into two incoherent terms: one in the interloper and the other one in perturbed autoionizing Rydberg series 1 . The incoherent decomposition (A6) of the resonance phase shift is compatible with Ueda's decomposition of cross sections (1) and (6) but holds for wider systems. For systems involving 2 closed and 1 open channels, both (A6) and (1) are exact. However, for systems with additional open channels, (A6) is still exact while (1) holds for each eigenchannel with an additional avoided crossing term with other eigenchannels.

Appendix B: Obtaining $\tilde{\mathbf{Z}}_{\text {res }}^{\mathrm{o}}$ at $\tilde{\varepsilon}_{1} \rightarrow \infty$.

For the short-range reactance matrix (7), in which $\tilde{K}^{\text {oo }}$ is a null matrix, the physical reactance matrix $\tilde{\mathbf{K}}$ is given by $-\tilde{K}^{\text {oc }}\left(\tan \tilde{\beta}+\tilde{K}^{\mathrm{cc}}\right)^{-1} \tilde{K}^{\mathrm{co}}$. For systems involving 2 closed channels, we may consider two column vectors, $\tilde{\xi}_{1}$ and $\tilde{\xi}_{2}$, defined in the open channel space by $\left\{\tilde{K}_{i 1} \mid i \in P\right\}$ and $\left\{\tilde{K}_{i 2} \mid i \in P\right\}$, respectively. Then $\tilde{K}^{\mathrm{oc}}$ can be written as $\left[\tilde{\xi}_{1} \tilde{\xi}_{2}\right]$ and

$$
\tilde{\mathbf{K}}=-\frac{1}{D_{\mathrm{cc}}}\left[\tilde{\xi}_{1} \tilde{\xi}_{1}^{\mathrm{T}} \tan \tilde{\beta}_{2}+\tilde{\xi}_{2} \tilde{\xi}_{2}^{\mathrm{T}} \tan \tilde{\beta}_{1}-\tilde{K}_{12}\left(\tilde{\xi}_{1} \tilde{\xi}_{2}^{\mathrm{T}}+\tilde{\xi}_{2} \tilde{\xi}_{1}^{\mathrm{T}}\right)\right]
$$

where $D_{\text {cc }}$ denotes $\operatorname{det}\left(\tan \tilde{\beta}+\tilde{K}^{\mathrm{cc}}\right)$. In the limit $\tan \tilde{\beta}_{1} \rightarrow \infty$, $\tilde{\mathbf{K}}$ becomes $-\tilde{\xi}_{2} \tilde{\xi}_{2}^{\mathrm{T}} / \tan \tilde{\beta}_{2}$ and its resonant solution is given by $\tilde{\xi}_{2}$, as can be confirmed by the following eigenvalue equation:

$$
\tilde{\mathbf{K}} \tilde{\xi}_{2}=-\frac{\tilde{\xi}_{2} \tilde{\xi}_{2}^{\mathrm{T}}}{\tan \tilde{\beta}_{2}} \tilde{\xi}_{2}=-\frac{\tilde{\xi}_{2}\left(\tilde{\xi}_{2}^{\mathrm{T}} \tilde{\xi}_{2}\right)}{\tan \tilde{\beta}_{2}}=-\frac{\tilde{\xi}_{2}^{2}}{\tan \tilde{\beta}_{2}} \tilde{\xi}_{2}=\tan \tilde{\delta}_{\mathrm{r} 2} \tilde{\xi}_{2}
$$

Appendix C: The $\tilde{K}_{23}=0$ case for the 3 channel systems involving 1 open channel

In the case of $\tilde{K}_{23}=0$, i.e., $\tilde{\xi}_{2}=0, \tilde{\varepsilon}_{2}, \tilde{q}_{2}$ and $\tilde{q}_{W}$ are singular, whereas $\tilde{q}_{1}$ remains finite. $\tilde{\varepsilon}_{2}$ cannot be defined because it is always infinite. The dynamic quantities that contain them require careful treatment. Two examples are as follows:

$$
\begin{aligned}
& \frac{\left(\tilde{\varepsilon}_{2}+\tilde{q}_{W}\right)^{2}}{\tilde{\varepsilon}_{2}^{2}+1} \underset{\tilde{\xi}_{2} \rightarrow 0}{\longrightarrow} 1 \\
& \tilde{W}_{\text {leff }} \underset{\tilde{\xi}_{2} \rightarrow 0}{\longrightarrow} \tilde{W}_{1} .
\end{aligned}
$$

Let us remove the singularities in $\tilde{\varepsilon}_{\text {leff }}$ and $\tilde{q}_{\text {leff }}$. For $\tilde{\varepsilon}_{\text {leff }}$, let us show the final result:

$$
\tilde{\varepsilon}_{1 \mathrm{eff}}=\tilde{\varepsilon}_{1}-\frac{\tilde{K}_{12}^{2}}{\tilde{W}_{1} \tan \tilde{\beta}_{2}}
$$

Next consider removing the singularities from $\tilde{q}_{\text {leff }}$. It is better to begin from its general equation:

$$
\tilde{q}_{1 \mathrm{eff}}=\frac{\tilde{q}_{1} \tilde{\varepsilon}_{2}^{2}+\left(\tilde{q}_{\mathrm{W}} \tilde{q}_{2}-1\right) \tilde{\varepsilon}_{2}+\tilde{q}_{1}-\tilde{q}_{2}-\tilde{q}_{\mathrm{W}}}{\left(\tilde{\varepsilon}_{2}+\tilde{q}_{2}\right)\left(\tilde{\varepsilon}_{2}+\tilde{q}_{\mathrm{W}}\right)} .
$$

Although all diverge, $\tilde{\varepsilon}_{2}+\tilde{q}_{2}$ and $\tilde{\varepsilon}_{2}+\tilde{q}_{\mathrm{w}}$ are dominated by $\tilde{\varepsilon}_{2}$ at the limit $\tilde{\xi}_{2} \rightarrow 0$ because the pole of $\tilde{\varepsilon}_{2}$ is of the second order whereas the others are of the first order. The limit is obtained as

$$
\tilde{q}_{1 \mathrm{eff}} \rightarrow \tilde{q}_{1}+\frac{\tilde{q}_{2} \tilde{q}_{\mathrm{W}}}{\tilde{\varepsilon}_{2}}=\tilde{q}_{1}+\frac{\tilde{K}_{12} \tilde{D}_{2}}{\tilde{\xi}_{1} \tilde{D}_{3} \tan \tilde{\beta}_{2}}
$$

Eq. (C1) yields the root of $\tilde{q}_{1 \text { eff }}$ as

$$
\tan \tilde{\beta}_{2}=\frac{\tilde{K}_{12} \tilde{D}_{2}}{\tilde{D}_{1}}
$$

From the results obtained thus far, the envelope becomes

$$
\tilde{\sigma}_{\text {interloper }} \underset{\tilde{\xi}_{2} \rightarrow 0}{\longrightarrow} \tilde{\sigma}_{0}
$$

and the perturbed spectrum of series 1 becomes

$$
\begin{aligned}
& \frac{\left(\tilde{\varepsilon}_{\text {leff }}+\tilde{q}_{\text {leff }}\right)^{2}}{\tilde{\varepsilon}_{\text {leff }}^{2}+1} \\
& =\frac{\left[\tilde{\varepsilon}_{1}+\frac{1}{\tilde{D}_{3} \tilde{W}_{1} \tan \tilde{\beta}_{2}}\left(-\tilde{\xi}_{1} \tilde{D}_{1} \tan \tilde{\beta}_{2}+\tilde{\xi}_{1} \tilde{K}_{12} \tilde{D}_{2}-\tilde{K}_{12}^{2} \tilde{D}_{3}\right)\right]^{2}}{\left(\tilde{\varepsilon}_{1}-\frac{\tilde{K}_{12}^{2}}{\tilde{W}_{1} \tan \tilde{\beta}_{2}}\right)^{2}+1}
\end{aligned}
$$

Previously, $\tilde{q}_{\text {leff }}$ was found to have two simple poles at $\tilde{q}_{W}$ 
and $\tilde{q}_{2}$. Note that their values are infinite in the present case and are reduced to one simple pole at 0 . The present analysis shows that the decrease in the number of simple poles from 2 to 1 causes a large change in the functional behavior of $\tilde{q}_{\text {leff }}$. Experimentally, whether $\tilde{K}_{23}$ is absolutely zero or almost zero may not be clearly determined. Since physics should not depend on small uncertainties in the measurement, a large change in the behavior of $\tilde{q}_{\text {leff }}$ should not affect the observables, such as autoionization cross sections. This might suggest that the cross sections are small at this troublesome region.

The above formulae cannot be used if $\tilde{D}_{3}=$ zero, which is the case for Fig. 3. In this case, the order of the poles of $\tilde{q}_{2}$ and $\tilde{q}_{\mathrm{w}}$ is 1 with respect to $\tilde{\xi}_{2}$, whereas that of $\tilde{\varepsilon}_{2}$ is 2 . This means that the numerator, $\tilde{\varepsilon}_{2}+\tilde{q}_{2}$, of the envelope is dominated by $\tilde{\varepsilon}_{2}$, and $\left(\tilde{\varepsilon}_{2}+\tilde{q}_{2}\right)^{2} /\left(\tilde{\varepsilon}_{2}^{2}+1\right)$ tends to 1 as $\tilde{\xi}_{2} \rightarrow 0$. In addition, the zero of $\tilde{D}_{3}$ should be taken into account. In this case, the envelope $\tilde{\sigma}_{\text {interloper }}$ cannot be defined as it is zero because $\tilde{D}_{3}=0 .\left|\tilde{D}_{3}\right|^{2}$ can be extracted from $\tilde{\sigma}_{\text {interloper }}$ and placed it into the numerator $\left(\tilde{\varepsilon}_{\text {leff }}+\tilde{q}_{\text {leff }}\right)^{2}$ of the perturbed dense spectra and then redefine $\tilde{q}_{\text {leff }}$ as $\tilde{D}_{3} \tilde{q}_{\text {leff }}$. Note that $\tilde{D}_{3} \tilde{\varepsilon}_{\text {leff }}=0$ while $\tilde{D}_{3} \tilde{q}_{\text {leff }}$ is not. Let us introduce the notation $q_{\text {leff }}^{\prime}$ for $\tilde{D}_{3} \tilde{q}_{\text {leff }}$. Then from $(\mathrm{C} 1)$,

$$
\tilde{q}_{\text {leff }}^{\prime}=-\frac{\tilde{D}_{1}}{\tilde{\xi}_{1}}+\frac{\tilde{D}_{2} \tilde{K}_{12}}{\tilde{\xi}_{1} \tan \tilde{\beta}_{2}}
$$

The reason for introducing the new notation is to keep the form of Ueda partitioning at least in appearance. The newly partitioned envelope was obtained by

$$
\tilde{\sigma}_{\text {interloper }}^{\prime}=\frac{4 \pi^{2} \alpha \omega}{3}
$$

\section{References}

1. Seaton, M. J. Rep. Prog. Phys. 1983, 46, 167.

2. Fano, U.; Rau, A. R. P. Atomic Collisions and Spectra; Academic: Orlando, U.S.A., 1986.

3. Giusti-Suzor, A.; Fano, U. J. Phys. B 1984, 17, 215.

4. Cooke, W. E.; Cromer, C. L. Phys. Rev. A 1985, 32, 2725.

5. Connerade, J. P.; Lane, A. M. Rep. Prog. Phys. 1988, 51, 1439.

6. Wintgen, D.; Friedrich, H. Phys. Rev. A 1987, 35, 1628.

7. Ueda, K. Phys. Rev. A 1987, 35, 2484.

8. Cho, B.; Lee, C.-W. Bull. Korean Chem. Soc. 2010, 31, 315.

9. Lee, C.-W. Bull. Korean Chem. Soc. 2010, 31, 1669.

10. Lecomte, J. M. J. Phys. B 1987, 20, 3645.

11. Cohen, S. Eur. Phys. J. D 1998, 4, 31.

12. Martins, M.; Zimmermann, P. Z. Phys. D 1993, 27, 115.

13. Lee, C.-W. Bull. Korean Chem. Soc. 2009, 30, 891.

14. Mies, F. H. Phys. Rev. 1968, 175, 164.

15. Fano, U.; Cooper, J. W. Phys. Rev. 1965, 137, A1364.

16. De Graaff, R. J.; Ubachs, W.; Hogervorst, W. Phys. Rev. A 1992, $45,166$.

17. Abutaleb, M.; De Graaff, R. J.; Ubachs, W.; Hogervorst, W. Phys. Rev. A 1991, 44, 4187.

18. Bente, E. A. J. M.; Hogervorst, W. J. Phys. B 1989, 22, 2679.

19. De Graaff, R. J.; Ubachs, W.; Hogervorst, W.; Abutaleb, M. Phys. Rev. A 1990, 42, 5473

20. Fano, U. Phys. Rev. 1961, 124, 1866. 